\title{
HOMOGENEOUS STATE FEEDBACK STABILIZATION OF HOMOGENEOUS SYSTEMS
}

\author{
LARS GRÜNE ${ }^{\dagger}$
}

\begin{abstract}
We show that for any asymptotically controllable homogeneous system in euclidian space (not necessarily Lipschitz at the origin) there exists a homogeneous control Lyapunov function and a homogeneous, possibly discontinuous state feedback law stabilizing the corresponding sampled closed loop system. If the system satisfies the usual local Lipschitz condition on the whole space we obtain semi-global stability of the sampled closed loop system for each sufficiently small fixed sampling rate, if the system satisfies a global Lipschitz condition we obtain global exponential stability for each sufficiently small fixed sampling rate. The control Lyapunov function and the feedback are based on the Lyapunov exponents of a suitable auxiliary system and admit a numerical approximation.
\end{abstract}

Key words. Homogeneous system, state feedback stabilization, control Lyapunov functions, Lyapunov exponents

AMS subject classifications. 93D15 (93D22, 93D30, 93D20)

1. Introduction. In this paper we consider the problem of state feedback stabilization of homogeneous control systems in $\mathbb{R}^{n}$. This problem has been considered by a number of authors during the last years, see e.g. [15, 16, 17, 20, 21, 22, 25], to mention just a few examples. Stability in this context will always mean asymptotic stability.

Homogeneous systems appear naturally as local approximations to nonlinear systems, which inherit some local properties of their homogeneous approximations, e.g. asymptotic controllability [14]. In order to make use of this property in the design of locally stabilizing feedbacks for nonlinear systems the main idea lies in the construction of homogeneous feedbacks, i.e. feedback laws that preserve homogenity for the resulting closed loop system. Utilizing a corresponding homogeneous Lyapunov function, those laws can then be shown to be locally stabilizing also for the approximated nonlinear system, cf. [14, 17, 19]. Regarding the existence of homogeneous stabilizing feedback laws, it was shown in [15] that if the system admits a continuous, but not necessarily homogeneous, stabilizing state feedback law, then there exists a homogeneous dynamic feedback stabilizing the system. Unfortunately, if we are looking for state feedback laws, it is in general not true that any continuously stabilizable homogeneous system is stabilizable by a continuous and homogeneous state feedback law, as the examples in [22] show. Even worse, there exist homogeneous systems, e.g. Brockett's classical example [2], which - although asymptotically controllable - do not admit a stabilizing continuous state feedback law at all.

Especially Brockett's results inspired the search for alternative feedback concepts. In the present paper we are going to use discontinuous state feedback laws for which the corresponding closed loop systems are defined as sampled systems. Although this is not a new concept, see e.g. [12, 13, 23], it has recently received new attention, see e.g. the survey [24]. In particular, it was shown in [4] that (global) asymptotic controllability is equivalent to the existence of a (globally) stabilizing discontinuous

\footnotetext{
${ }^{\dagger}$ Fachbereich Mathematik, J.W. Goethe-Universität, Postfach 111932, 60054 Frankfurt a.M., Germany, E-Mail: gruene@math.uni-frankfurt.de. This paper has been written while the author was visiting the Dipartimento di Matematica, Universitá di Roma "La Sapienza", Italy, supported by DFG-Grant GR1569/2-1.
} 
state feedback law for the sampled closed loop system. Stability in this context means asymptotic stability for the sampled trajectories, where-in general - the intersampling times have to tend to zero close to the equilibrium and far away from it. A related but slightly different concept of a discontinuous feedback is the notion of discrete feedback introduced in [7]; here also sampled trajectories are considered, but with fixed intersampling times. With this approach it was possible to show in [10] that for semilinear systems asymptotic controllability is equivalent to (exponential) discrete feedback stabilizability.

The goal of the present paper is to provide a link between these two concepts in the framework of homogeneous systems. As in [10] we use a spectral characterization of asymptotic controllability by means of Lyapunov exponents, and obtain stability results for fixed sampling rates; as in [4] we construct the feedback based on a suitable (and here also homogeneous) control Lyapunov function, and obtain stability not only for fixed intersampling times but for all sufficiently small ones. Furthermore, and this is a key feature of our construction, the resulting stabilizing state feedback law is homogeneous, thus rendering the corresponding closed loop system homogeneous. All this will be done just under the assumption that the corresponding homogeneous system is asymptotically controllable.

The organization of this paper is as follows. After defining the setup and the concepts we pursue, in Section 3 we introduce a class of auxiliary systems we call homogeneous-in-the-state. In some sense these systems have a built in homogenity for each control value. These systems will be simplifyed by suitable coordinate and time transformations, and for the resulting system we will characterize asymptotic controllability by means of its Lyapunov exponents. In Section 4 we will use this characterization in order to construct a suitable control Lyapunov function which will then be used for the construction of the stabilizing feedback law. After giving some hints about a numerical approximation of these feedback laws in Section 5, we will return to the homogeneous systems in Section 6 and prove the stabilization result by showing that these systems can easily be transformed into systems homogeneous-inthe-state without loosing the asymptotic controllability property. Finally, in Section 7 we discuss two examples.

2. Setup. We consider a class of systems

$$
\dot{x}(t)=g(x(t), w(t))
$$

on $\mathbb{R}^{n}$ where $w(\cdot) \in \mathcal{W}$, and $\mathcal{W}$ denotes the space of measurable and locally essentially bounded functions from $\mathbb{R}$ to $W \subset \mathbb{R}^{m}$. We assume that the vector field $g$ is continuous, $g(\cdot, w)$ is locally Lipschitz on $\mathbb{R}^{n} \backslash\{0\}$ for each $w \in W$, and satisfies the following property.

DEFINITION 2.1. We call $g$ homogeneous if there exist $r_{i}>0, i=1, \ldots, n$, $s_{j}>0, j=1, \ldots, m$ and $\tau \in\left(-\min _{i} r_{i}, \infty\right)$ such that

$$
g\left(\Lambda_{\alpha} x, \Delta_{\alpha} w\right)=\alpha^{\tau} \Lambda_{\alpha} g(x, w) \text { for all } w \in W, \alpha \geq 0
$$

where

$$
\Lambda_{\alpha}=\left(\begin{array}{cccc}
\alpha^{r_{1}} & 0 & \ldots & 0 \\
0 & \ddots & \ddots & \vdots \\
\vdots & \ddots & \ddots & 0 \\
0 & \ldots & 0 & \alpha^{r_{n}}
\end{array}\right) \quad \text { and } \quad \Delta_{\alpha}=\left(\begin{array}{cccc}
\alpha^{s_{1}} & 0 & \ldots & 0 \\
0 & \ddots & \ddots & \vdots \\
\vdots & \ddots & \ddots & 0 \\
0 & \ldots & 0 & \alpha^{s_{m}}
\end{array}\right)
$$


are called dilation matrices. With $k=\min _{i} r_{i}$ we denote the minimal power (of the state dilation) and the value $\tau \in(-k, \infty)$ is called the degree of the system.

This definition generalizes the one given in [22] to the case of a multidimensional control input, see e.g. cite [14] for an alternative definition (equivalent on $\mathbb{R}^{n}$ ) for vector fields on arbitrary manifolds. The use of dilation matrices instead of the usual dilation functions allows a more compact notation in what follows. Observe that $g$ is Lipschitz in the origin iff $\tau \geq 0$ and globally Lipschitz iff $\tau=0$, furthermore the definition implies $g(0,0)=0$.

Corresponding to the dilation matrix $\Lambda_{\alpha}$ we define a function $N: \mathbb{R}^{n} \rightarrow[0, \infty)$ which can be interpreted as a "dilated norm" w.r.t. $\Lambda_{\alpha}$. Denoting $d=2 \prod_{i=1}^{n} r_{i}$ we define $N(x)$ by

$$
N(x):=\left(\sum_{i=1}^{n} x_{i}^{\frac{d}{r_{i}}}\right)^{\frac{1}{d}}
$$

implying $N(0)=0, N(x)>0$ if $x \neq 0$, and $N\left(\Lambda_{\alpha} x\right)=\alpha N(x)$.

Note that the trajectories of (2.1) may tend to infinity in finite time if $\tau>0$ and that uniqueness of the trajectory may not hold if $\tau<0$, however it holds away from the origin. As long as uniqueness holds (i.e. if $\tau \geq 0$ or the trajectory does not cross the origin) we denote the (open loop) trajectories of $(2.1)$ by $x\left(t, x_{0}, w(\cdot)\right)$ for each $x_{0} \in \mathbb{R}^{n}$ and each $w(\cdot) \in \mathcal{W}$, where $x\left(0, x_{0}, w(\cdot)\right)=x_{0}$, Then from Definition 2.1 we obtain

$$
x\left(t, \Lambda_{\alpha} x_{0}, \Delta_{\alpha} w\left(\alpha^{\tau} \cdot\right)\right)=\Lambda_{\alpha} x\left(\alpha^{\tau} t, x_{0}, w(\cdot)\right)
$$

for $x_{0} \in \mathbb{R}^{n}$. If uniqueness fails to hold $x\left(\cdot, x_{0}, w(\cdot)\right)$ shall denote one possible trajectory; in this case we implicitely assume the following definitions to be valid for all possible trajectories.

The following definition gives the meaning of asymptotic controllability.

Definition 2.2. We call the system asymptotically controllable (to the origin), if for each $x_{0} \in \mathbb{R}^{n}$ there exists $w_{x_{0}}(\cdot) \in \mathcal{W}$ such that $\left\|x\left(t, x_{0}, w_{x_{0}}(\cdot)\right)\right\| \rightarrow 0$ as $t \rightarrow \infty$.

We now discuss the concept of homogeneous state feedbacks. A state feedback law is a map $F: \mathbb{R}^{n} \rightarrow W$. A homogeneous state feedback law satisfies $F\left(\Lambda_{\alpha} x\right)=\Delta_{\alpha} F(x)$ for all $x \in \mathbb{R}^{n}$ and all $\alpha \geq 0$, thus implying $g\left(\Lambda_{\alpha} x, F\left(\Lambda_{\alpha} x\right)\right)=\alpha^{t} \Lambda_{\alpha} g(x, F(x))$, i.e. the closed loop system using $F$ becomes homogeneous. Observe that $W$ needs to satisfy some structural condition in order to allow nontrivial homogeneous feedbacks; in what follows we will assume

$$
\Delta_{\alpha} W \subset W \text { for all } \alpha \geq 0, \text { where } \Delta_{\alpha} W:=\left\{\Delta_{\alpha} w \mid w \in W\right\}
$$

which gives a necessary and sufficient condition for the fact that given some $c>0$ any homogeneous map $F: \mathbb{R}^{n} \rightarrow \mathbb{R}^{m}$ satisfying $F(x) \in W$ on $\left\{x \in \mathbb{R}^{d} \mid N(x)=c\right\}$ satisfies $F(x) \in W$ for all $x \in \mathbb{R}^{n}$.

Note that we do not require any continuity property of $F$. This is due to the fact, that in many examples stabilizing continuous feedbacks cannot exist, cf. e.g. [24, Section 2.2] where also Brockett's classical example [2] is discussed which-in suitable coordinates - is in fact a homogeneous system. Furthermore, even if stabilizing continuous feedback laws exist, it is possible that no such law is homogeneous, as the examples in [22] show (Brockett's example and the first example from [22] will be discussed in Section 7). However, using discontinuous feedbacks for the solutions 
of the classical closed loop system $\dot{x}=g(x, F(x))$ the usual existence and uniqueness results might not hold. In order to obtain a meaningful solution for the closed loop system we use the following concept of a sampled closed loop system.

Definition 2.3. (Sampled closed loop system) Consider a feedback law $F: \mathbb{R}^{n} \rightarrow$ $W$. An infinite sequence $\pi=\left(t_{i}\right)_{i \in \mathbb{N}_{0}}$ of times satisfying

$$
0=t_{0}<t_{1}<t_{2}<\ldots \quad \text { and } \quad t_{i} \rightarrow \infty \text { as } i \rightarrow \infty
$$

is called a sampling schedule. The values

$$
\Delta t_{i}:=t_{i+1}-t_{i} \quad \text { and } \quad d(\pi):=\sup _{i \in \mathbb{N}_{0}} \Delta t_{i}
$$

is called the intersampling times and the sampling rate, respectively. For any sampling schedule $\pi$ the corresponding sampled or $\pi$-trajectory $x_{\pi}\left(t, x_{0}, F\right)$ with initial value $x_{0} \in \mathbb{R}^{n}$ at initial time $t_{0}=0$ is defined inductively by

$$
x_{\pi}\left(t, x_{0}, F\right)=x\left(t-t_{i}, x_{i}, F\left(x_{i}\right)\right), \text { for all } t \in\left[t_{i}, t_{i+1}\right], i \in \mathbb{N}_{0}
$$

where $x_{i}=x_{\pi}\left(t_{i}, x_{0}, F\right)$ and $x\left(t, x_{i}, F\left(x_{i}\right)\right)$ denotes the (open loop) trajectory of (2.1) with constant control value $F\left(x_{i}\right)$ and initial value $x_{i}$.

Observe that this definition guarantees the existence and uniqueness of trajectories in positive time on their maximal intervals of existence (except possibly at the origin if $\tau<0$, in which case we use the same convention as for open loop trajectories). Moreover, the sampled $\pi$-trajectories have a meaningful physical interpretation, as they correspond to an implementation of the feedback law $F$ using a digital controller.

The next definition introduces control Lyapunov functions which will be vital for the construction of the feedback.

Definition 2.4. A continuous function $V: \mathbb{R}^{n} \rightarrow[0, \infty)$ is called a control Lyapunov function (clf), if it is positive definite (i.e. $V(0)=0$ iff $V=0$ ), proper (i.e. $V(x) \rightarrow \infty$ as $\|x\| \rightarrow \infty)$, and there exists a continuous and positive definite function $P: \mathbb{R}^{n} \rightarrow[0, \infty)$ such that for each bounded subset $G \subset \mathbb{R}^{n}$ there exists a compact subset $W_{G} \subset W$ with

$$
\min _{v \in \operatorname{cog}\left(x, W_{G}\right)} D V(x ; w) \leq-P(x) \text { for all } x \in G
$$

Here $D V(x ; v)$ denotes the lower directional derivative

$$
D V(x ; v):=\liminf _{t \searrow 0, v^{\prime} \rightarrow v} \frac{1}{t}\left(V\left(x+t v^{\prime}\right)-V(x)\right)
$$

$g\left(x, W_{G}\right):=\left\{g(x, w) \mid w \in W_{G}\right\}$, and $\operatorname{cog}\left(x, W_{G}\right)$ denotes the convex hull of $g\left(x, W_{G}\right)$.

The following definition now describes the stability concepts we will use in this paper. For this definition recall that a function $\gamma:[0, \infty) \rightarrow[0, \infty)$ is of class $\mathcal{K}$, if it satisfies $\gamma(0)=0$ and is continuous and strictly increasing, and a function $\beta$ : $[0, \infty)^{2} \rightarrow[0, \infty)$ is of class $\mathcal{K} \mathcal{L}$, if it is decreasing to zero in the second and of class $\mathcal{K}$ in the first argument.

DEFinition 2.5. We call the sampled closed loop system from Definition 2.3

(i) semi-globally practically stable with fixed sampling rate, if there exists a class 
$\mathcal{K} \mathcal{L}$ function $\beta$ such that for each open set $B \subset \mathbb{R}^{n}$ and each compact set $K \subset \mathbb{R}^{n}$ satisfying $0 \in B \subset K$ there exists $h>0$ such that

$$
x_{\pi}\left(t, x_{0}, F\right) \notin B \Rightarrow\left\|x_{\pi}\left(t, x_{0}, F\right)\right\| \leq \beta\left(\left\|x_{0}\right\|, t\right)
$$

for all $x_{0} \in K$ and all $\pi$ with $d(\pi) \leq h$,

(ii) semi-globally stable with fixed sampling rate, if (i) holds and the sampling rate $h>0$ can be chosen independently of $B$,

(iii) globally practically stable with fixed sampling rate if (i) holds and the sampling rate $h>0$ can be chosen independently of $K$,

(iv) globally stable with fixed sampling rate if (i) holds and the sampling rate $h>0$ can be chosen independently of $K$ and $B$.

We call the stability in (i)-(iv) exponential if $\beta$ can be chosen as $C e^{-\sigma t}\left\|x_{0}\right\|$ for constants $C, \sigma>0$ which may depend on $K$, and uniformly exponential if $C, \sigma>0$ can be chosen independently of $K$.

Note that each of the concepts (ii)-(iv) implies (i) which is equivalent to the sstability property as defined in [4], cf. also [24, Sections 3.1 and 5.1]. Hence any of these concepts implies global stability for the (possibly nonunique) limiting trajectories as $h \rightarrow 0$. The difference "only" lies in the performance with fixed sampling rate. From the applications point of view, however, this is an important issue, since e.g. for an implementation of a feedback using some digital controller arbitrary small sampling rates in general will not be realizable. Furthermore if the sampling rate tends to zero the resulting stability may be sensitive to measurement errors, if the feedback is based on a non-smooth clf, see $[18,24]$. In contrast to this it is quite straightforward to see that for a fixed sampling rate the stability is in fact robust to small errors in the state measurement (small, of course, relative to the norm of the current state of the system) if the corresponding clf is Lipschitz, cf. [24, Theorem E].

The main result we will prove in this paper is the following theorem on the existence of a homogeneous clf $V$ and a homogeneous stabilizing feedback $F$.

Theorem 2.6. Consider system (2.1) satisfying Definition 2.1 with dilation matrices $\Lambda_{\alpha}$ and $\Delta_{\alpha}$, minimal power $k>0$, and degree $\tau \in(-k, \infty)$, and assume asymptotic controllability. Then there exists $\mu>0$ and a clf $V$ being Lipschitz on $\mathbb{R}^{n} \backslash\{0\}$, satisfying

$$
V\left(\Lambda_{\alpha}(x)\right)=\alpha^{2 k} V(x)
$$

and

$$
\min _{v \in \operatorname{cog}\left(x, W_{x}\right)} D V_{\beta}(x ; v) \leq-2 \mu N^{\tau}(x) V_{\beta}(x)
$$

for the function $N$ from (2.3) and $W_{x}=\Delta_{N(x)} U$ for some suitable compact subset $U \subset W$.

Furthermore there exists a feedback law $F: \mathbb{R}^{n} \rightarrow W$ satisfying $F(x) \in W_{x}$ and $F\left(\Lambda_{\alpha} x\right)=\Delta_{\alpha} F(x)$ for all $x \in \mathbb{R}^{n}$ and all $\alpha \geq 0$ such that the corresponding sampled closed loop system is either

(i) semi-globally stable (if $\tau>0$ ), or

(ii) globally uniformly exponentially stable (if $\tau=0$ ), or

(iii) globally practically exponentially stable (if $\tau<0$ )

with fixed sampling rate.

The proof is given in Section 6 . 
3. Systems homogeneous-in-the-state. In this section we define a class of auxiliary systems which are homogeneous in the state and will turn out to be useful for our analysis. By suitable coordinate and time transformations we will then simplify this class of systems and and characterize controllability of the simplified system by means of its Lyapunov exponents.

We consider the class of systems

$$
\dot{x}(t)=f(x(t), u(t))
$$

on $\mathbb{R}^{n}$ where $u(\cdot) \in \mathcal{U}$, and $\mathcal{U}$ denotes the space of measurable functions from $\mathbb{R}$ to some compact set $U \subset \mathbb{R}^{m}$. We assume that the vector field $f$ is continuous, $f(\cdot, u)$ is locally Lipschitz on $\mathbb{R}^{n} \backslash\{0\}$ for each $u \in U$, and satisfies the following property.

DEFINITION 3.1. We call $f$ homogeneous-in-the-state if there exist $r_{i}>0, i=$ $1, \ldots, n$ and $\tau \in\left(-\min _{i} r_{i}, \infty\right)$ such that

$$
f\left(\Lambda_{\alpha} x, u\right)=\alpha^{\tau} \Lambda_{\alpha} f(x, u) \text { for all } u \in U
$$

where $\Lambda_{\alpha}$ is the dilation matrix as in Definition 2.1, $k=\min _{i} r_{i}$ is called the minimal power and the value $\tau \in(-k, \infty)$ is called the degree of the system.

Note that this definition implies $f(0, u)=0$ for all $u \in U$. We denote the trajectories of (3.1) with initial value $x_{0}$ at the time $t=0$ and control function $u(\cdot) \in \mathcal{U}$ again by $x\left(t, x_{0}, u(\cdot)\right)$. Observe that also the trajectories of (3.1) may escape in finite time if $\tau>0$ and that uniqueness of the trajectory may not hold in the origin if $\tau<0$ (here again we use the convention as for the trajectories of (2.1)). As long as the trajectories exist and uniqueness holds we obtain from Definition 3.1 that

$$
x\left(t, \Lambda_{\alpha} x_{0}, u\left(\alpha^{\tau} \cdot\right)\right)=\Lambda_{\alpha} x\left(\alpha^{\tau} t, x_{0}, u(\cdot)\right)
$$

for all $x_{0} \in \mathbb{R}^{n}$.

Besides being useful auxiliary systems for our stabilization problem for homogeneous systems, homogeneous-in-the-state systems themselves form an interesting class of systems. They generalize homogeneous bilinear and semilinear systems (see e.g. $[5,6,7,10])$. Generally speaking they model systems in which the control affects parameters of the system rather that representing some force acting on the system, cf. the examples in [8,9]. Also for this class of systems there exist examples which are stabilizable but not with a continuous feedback law, see [24, Example after Theorem A]. Note that this class can be generalized analogously to the generalization of semilinear systems made in [10]; all results in this paper can easily be adapted to that case.

Applying suitable coordinate and time transformations we can considerably simplify the class of systems to be considered: Using the dilated norm $N$ from (2.3) the function

$$
P(x):=\Lambda_{N(x)}^{-1} x
$$

defines a projection from $\mathbb{R}^{n} \backslash\{0\}$ onto $N^{-1}(1)$ satisfying $P\left(\Lambda_{\alpha} x\right)=P(x)$ for all $\alpha>0$. We denote the $n-1$ dimensional embedded unit sphere $\left\{x \in \mathbb{R}^{n} \mid\|x\|=1\right\}$ by $\mathbb{S}^{n-1}$. Then, since $N(t x)$ is strictly increasing in $t \geq 0$ the function $S: N^{-1}(1) \rightarrow \mathbb{S}^{n-1}$, $S(x)=x /\|x\|$ is a diffeomorphism between these two manifolds, thus we can define a coordinate transformation $y=\Psi(x)$ by

$$
\Psi(x)=N(x)^{k} S(P(x)), \quad \Psi^{-1}(y)=\Lambda_{\sqrt[k]{\|y\|}} S^{-1}\left(\frac{y}{\|y\|}\right)
$$


and $\Psi(0)=0, \Psi^{-1}(0)=0$, which is continuous on $\mathbb{R}^{n}$ and $C^{1}$ on $\mathbb{R}^{n} \backslash\{0\}$. This definition implies

$$
\Psi\left(\Lambda_{\alpha} x\right)=\alpha^{k} \Psi(x), \quad \Psi^{-1}\left(\alpha^{k} y\right)=\Lambda_{\alpha} \Psi^{-1}(y)
$$

and by differentiation of $\Psi\left(\Lambda_{\alpha} x\right)$ and $\alpha^{k} \Psi(x)$ one sees

$$
D \Psi\left(\Lambda_{\alpha} x\right)=\alpha^{k} \Lambda_{\alpha}^{-1} D \Psi(x) .
$$

Thus defining

$$
\tilde{f}(y, u)=D \Psi\left(\Psi^{-1}(y)\right) f\left(\Psi^{-1}(y), u\right)
$$

we obtain (with $x=\Psi^{-1}(y)$ )

$$
\tilde{f}\left(\alpha^{k} y, u\right)=D \Psi\left(\Lambda_{\alpha} x\right) f\left(\Lambda_{\alpha} x, u\right)=\alpha^{k} \Lambda_{\alpha}^{-1} D \Psi(x) \alpha^{\tau} \Lambda_{\alpha} f(x, u)=\alpha^{\tau} \alpha^{k} \tilde{f}(y, u)
$$

implying

$$
\tilde{f}(\alpha y, u)=\alpha^{\gamma+1} \tilde{f}(y, u),
$$

with $\gamma=\tau / k$, i.e. $\tilde{f}$ is homogeneous-in-the-state with respect to the standard dilation $\Lambda_{\alpha}=\alpha \mathrm{Id}$, with mimimal power $k=1$, and with degree $\tau=\gamma$.

Furthermore setting $\bar{f}(y, u)=\tilde{f}(y, u)\|y\|^{-\gamma}$ (which defines a time transformation for $\tilde{f}$ ) we obtain a system with degree $\tau=0$. In what follows we will therefore assume

$$
f(\alpha x, u)=\alpha f(x, u) \text { for all } x \in \mathbb{R}^{n}, \alpha \geq 0
$$

and will retranslate the results to the general case in Theorem 4.3. Observe that the new $f$ is now globally Lipschitz with a uniform constant which we will denote by $L$.

In order to obtain a way to characterize asymptotic controllability of (3.4) we introduce the finite time exponential growth rate (cf. $[10,11]$ )

$$
\lambda^{t}\left(x_{0}, u(\cdot)\right)=\frac{1}{t} \ln \frac{\left\|x\left(t, x_{0}, u(\cdot)\right)\right\|}{\left\|x_{0}\right\|} .
$$

It follows immediately from (3.4) that $x\left(t, \alpha x_{0}, u(\cdot)\right)=\alpha x\left(t, x_{0}, u(\cdot)\right)$ and thus the growth rates satisfy $\lambda^{t}\left(x_{0}, u(\cdot)\right)=\lambda^{t}\left(\alpha x_{0}, u(\cdot)\right)$ for all $x_{0} \in \mathbb{R}^{d} \backslash\{0\}$ and all $\alpha>0$. The meaning of $\lambda^{t}$ is described by the following proposition.

PROPOSITION 3.2. System (3.4) is asymptotically controllable if and only if there exists a time $T>0$ and some $\rho>0$ such that for each $x \in \mathbb{R}^{n} \backslash\{0\}$ there exists $u_{x}(\cdot) \in \mathcal{U}$ with

$$
\lambda^{t}\left(x, u_{x}(\cdot)\right)<-\rho \text { for all } t \geq T
$$

Proof. Obviously (3.5) implies exponential controllability, thus in particular asymptotic controllability.

For the converse implication since $\lambda^{t}(x, u(\cdot))=\lambda^{t}(\alpha x, u(\cdot))$ it is sufficient to show (3.5) for $\|x\|=1$, i.e. $x \in \mathbb{S}^{n-1}$. Asymptotic controllability implies that for each $x \in \mathbb{S}^{n-1}$ there exist $\tilde{u}_{x}(\cdot) \in \mathcal{U}, \tilde{t}_{x}>0$, and $C_{x}>0$ such that $\left\|\varphi\left(\tilde{t}_{x}, x, \tilde{u}_{x}(\cdot)\right)\right\|<1 / 2$, and $\left\|\varphi\left(t, x, \tilde{u}_{x}(\cdot)\right)\right\|<C_{x}$ for all $t \in\left[0, \tilde{t}_{x}\right]$. By compactness of $\mathbb{S}^{n-1}$ and continuous dependence on the initial value we can choose the controls such that $T_{1}=\sup _{x \in \mathbb{S}^{n-1}} \tilde{t}_{x}$ 
and $C=\sup _{x \in \mathbb{S}^{n-1}} C_{x}$ are finite. Now for each $x \in \mathbb{S}^{n-1}$ we define $u_{x}(\cdot)$ and a sequence $t_{i}$ inductively by $t_{0}=0$ and

$$
t_{i+1}=t_{i}+\tilde{t}_{x_{i}}, \quad u_{x}(t)=\tilde{u}_{x_{i}}\left(t-t_{i}\right), t \in\left[t_{i}, t_{i+1}\right]
$$

where $x_{i}=\varphi\left(t_{i}, x, u_{x}(\cdot)\right) /\left\|\varphi\left(t_{i}, x, u_{x}(\cdot)\right)\right\|$. Choosing $t_{i}$ maximal with $t_{i} \leq t$ (i.e. $t-t_{i}<T_{1}$ and $\left.t_{i}>t-T_{1}\right)$ this implies

$$
\lambda^{t}\left(x, u_{x}(\cdot)\right)=\frac{t_{i}}{t} \lambda^{t_{i}}\left(x, u_{x}(\cdot)\right)+\frac{t-t_{i}}{t} \lambda^{t-t_{i}}\left(x_{i}, u_{x}\left(t_{i}+\cdot\right)\right) \leq \frac{t-T_{1}}{t} \ln \frac{1}{2}+\frac{T_{1}}{t} \ln C
$$

where the last expression is independent of $x$ and negative for all $t \geq T$ for $T>0$ sufficiently large, which yields the assertion.

In fact, we can show something more than just the negativity of the finite time exponential growth rates. We define the Lyapunov exponent of each trajectory by

$$
\lambda(x, u(\cdot)):=\limsup _{t \rightarrow \infty} \lambda^{t}(x, u(\cdot))
$$

and the supremum w.r.t. the state and infimum w.r.t. the control over these exponents by

$$
\sigma:=\sup _{x \in \mathbb{R}^{n} \backslash\{0\}} \inf _{u(\cdot) \in \mathcal{U}} \lambda(x, u(\cdot)) .
$$

Lyapunov exponents for control systems have been utilized in the analysis of bilinear systems (see e.g. [5] for some basic concepts and [6] for a detailled exposition) and for the global stabilization of semilinear and the local stabilization of differentiable nonlinear systems at singular points [10]. In the homogeneous setup we obtain the following characterization.

Proposition 3.3. Consider the system (3.4) and its sup-inf Lyapunov exponent $\sigma$. Then for each $\rho \in(0, \sigma)$ there exists $T>0$ such that for each $x \in \mathbb{R}^{n} \backslash\{0\}$ there exists $u_{x}(\cdot) \in \mathcal{U}$ with

$$
\lambda^{t}\left(x, u_{x}(\cdot)\right)<-\rho \text { for all } t \geq T
$$

Proof. Exactly as [10, Proof of Proposition 3.4].

Since by Proposition 3.2 for our class (3.4) of homogeneous systems asymptotic controllability immediately implies $\sigma<0$, Proposition 3.3 establishes a spectral condition for the asymptotic controllability of (3.4).

4. Stabilization of systems homogeneous-in-the-state. In this section we will construct a Lyapunov function and a stabilizing feedback for system (3.4). Afterwards we retranslate this stabilization result to general systems homogeneous-inthe-state from Definition 3.1.

We begin with the construction of a homogeneous Lyapunov function for system (3.4). First observe that the projection

$$
s\left(t, s_{0}, u(\cdot)\right):=\frac{x\left(t, x_{0}, u(\cdot)\right)}{\left\|x\left(t, x_{0}, u(\cdot)\right)\right\|}, \quad s_{0}=\frac{x_{0}}{\left\|x_{0}\right\|}
$$

of (3.4) onto $\mathbb{S}^{n-1}$ is well defined due to the homogenity of the system. A simple application of the chain rule shows that $s$ is the solution of

$$
\dot{s}(t)=f_{\mathbb{S}}(s(t), u(t)), \quad f_{\mathbb{S}}(s, u)=f(s, u)-\langle s, f(s, u)\rangle s
$$


and that for $s_{0}=x_{0} /\left\|x_{0}\right\|$ the exponential growth rate $\lambda^{t}$ satisfies

$$
\lambda^{t}\left(x_{0}, u(\cdot)\right)=\lambda^{t}\left(s_{0}, u(\cdot)\right)=\frac{1}{t} \int_{0}^{t} q\left(s\left(\tau, s_{0}, u(\cdot)\right), u(\tau)\right) d \tau
$$

with $q(s, u)=\langle s, f(s, u)\rangle$. Thus defining the discounted integral

$$
J_{\delta}\left(s_{0}, u(\cdot)\right):=\int_{0}^{\infty} e^{-\delta \tau} q\left(s\left(\tau, s_{0}, u(\cdot)\right), u(\tau)\right) d \tau
$$

and the corresponding optimal value function

$$
v_{\delta}\left(s_{0}\right):=\inf _{u(\cdot) \in \mathcal{U}} J_{\delta}\left(s_{0}, u(\cdot)\right)
$$

from Propositions 3.2 and 3.3, and [10, Lemma 3.5(ii)] we obtain that if system (3.4) is asymptotically controllable then for each $\rho \in(0, \sigma)$ there exists $\delta_{\rho}>0$ such that for all $\delta \in\left(0, \delta_{\rho}\right]$ and all $s_{0} \in \mathbb{S}^{n-1}$ the inequality

$$
\delta v_{\delta}\left(s_{0}\right)<-\rho
$$

holds. Note that $v_{\delta}$ is Hölder continuous and bounded for each $\delta>0$, cp. e.g. [1]. We now fix some $\rho \in(0, \sigma)$ and some $\delta \in\left(0, \delta_{\rho}\right]$ and define

$$
V_{0}(x):=e^{2 v_{\delta}(x /\|x\|)}\|x\|^{2} .
$$

Lemma 4.1. The function $V_{0}$ is a clf which is homogeneous with degree 1 (with respect to the standard dilation) and satisfies

$$
\min _{v \in \operatorname{co} f(x, U)} D V_{0}(x ; v) \leq-2 \rho V_{0}(x) .
$$

Proof. Homogeneity, positive definiteness and properness follow immediately from the definition. Now for each $t>0$ the function $v_{\delta}$ satisfies the dynamic programming principle

$$
v_{\delta}\left(s_{0}\right)=\inf _{u(\cdot) \in \mathcal{U}}\left\{\int_{0}^{t} e^{-\delta \tau} q\left(s\left(\tau, s_{0}, u(\cdot)\right), u(\tau)\right) d \tau+e^{-\delta t} v_{\delta}\left(s\left(t, s_{0}, u(\cdot)\right)\right)\right\},
$$

see e.g. [1]. Abbreviating $q\left(t, s_{0}, u(\cdot)\right)=q\left(s\left(t, s_{0}, u(\cdot)\right), u(t)\right)$ and using $e^{-\delta t}-1 \geq-\delta t$ we obtain for the integral part of this equality

$$
\int_{0}^{t} e^{-\delta \tau} q\left(\tau, s_{0}, u(\cdot)\right) d \tau \geq \int_{0}^{t} q\left(\tau, s_{0}, u(\cdot)\right)+\left(e^{-\delta t}-1\right) M_{q} d \tau=t \lambda^{t}\left(s_{0}, u(\cdot)\right)-M_{q} \delta \frac{t^{2}}{2}
$$

where $M_{q}$ denotes a bound of $|q|$. Thus with $s_{0}=x_{0} /\left\|x_{0}\right\|$ we obtain

$$
\begin{aligned}
V_{0}\left(x_{0}\right) & \geq \inf _{u(\cdot) \in \mathcal{U}} \exp \left[2 t \lambda^{t}(x, u(\cdot))-M_{q} \delta t^{2}+2 e^{-\delta t} v_{\delta}\left(s\left(t, s_{0}, u(\cdot)\right)\right)\right]\left\|x_{0}\right\|^{2} \\
& =\inf _{u(\cdot) \in \mathcal{U}} e^{2 t \lambda^{t}(x, u(\cdot))} e^{-M_{q} \delta t^{2}} e^{2\left(e^{-\delta t}-1\right) v_{\delta}\left(s\left(t, s_{0}, u(\cdot)\right)\right)} e^{2 v_{\delta}\left(s\left(t, s_{0}, u(\cdot)\right)\right)}\left\|x_{0}\right\|^{2} \\
& =\inf _{u(\cdot) \in \mathcal{U}} \frac{\left\|x\left(t, x_{0}, u(\cdot)\right)\right\|^{2}}{\left\|x_{0}\right\|^{2}} e^{-M_{q} \delta t^{2}} e^{2\left(e^{-\delta t}-1\right) v_{\delta}\left(s\left(t, s_{0}, u(\cdot)\right)\right)} e^{2 v_{\delta}\left(s\left(t, s_{0}, u(\cdot)\right)\right)}\left\|x_{0}\right\|^{2} \\
& =\inf _{u(\cdot) \in \mathcal{U}} e^{-M_{q} \delta t^{2}+2\left(e^{-\delta t}-1\right) v_{\delta}\left(s\left(t, s_{0}, u(\cdot)\right)\right)} V_{0}\left(x\left(t, x_{0}, u(\cdot)\right)\right) \\
& \geq \inf _{u(\cdot) \in \mathcal{U}} e^{-M_{q} \delta t^{2}+2\left(1-e^{-\delta t}\right) \rho / \delta} V_{0}\left(x\left(t, x_{0}, u(\cdot)\right)\right) .
\end{aligned}
$$


Now for each $t>0$ we choose $u_{t}(\cdot) \in \mathcal{U}$ such that the infimum of the last expression is attained up to $t^{2}$. Using $b-b^{2} \leq 1-e^{-b} \leq b$ for $b>0$ we can conclude

$$
\begin{aligned}
V_{0}\left(x\left(t, x_{0}, u_{t}(\cdot)\right)\right)-V_{0}\left(x_{0}\right) & \leq\left(1-e^{-M_{q} \delta t^{2}+2\left(1-e^{-\delta t}\right) \rho / \delta}\right) V_{0}\left(x\left(t, x_{0}, u_{t}(\cdot)\right)\right)+t^{2} \\
& \leq\left(1-e^{-M_{q} \delta t^{2}+2 t \rho-2 \delta t^{2} \rho}\right) V_{0}\left(x\left(t, x_{0}, u_{t}(\cdot)\right)\right)+t^{2} \\
& \leq\left(-2 t \rho+\left(M_{q}+2 \rho\right) \delta t^{2}\right) V_{0}\left(x\left(t, x_{0}, u(\cdot)\right)\right)+t^{2}
\end{aligned}
$$

for all $t>0$ sufficiently small. Denoting $v_{t}=\left(x\left(t, x_{0}, u_{t}(\cdot)\right)-x_{0}\right) / t$ we obtain

$$
\frac{1}{t}\left(V_{0}\left(x_{0}+t v_{t}\right)-V_{0}\left(x_{0}\right)\right) \leq-2 \rho V_{0}\left(x\left(t, x_{0}, u(\cdot)\right)\right)+\left(M_{q}+2 \rho\right) \delta t V_{0}\left(x\left(t, x_{0}, u(\cdot)\right)\right)+t
$$

and since by compactness of $U$ there exists a $v \in \operatorname{cof}(x, U)$ and a sequence $t_{i} \rightarrow 0$ such that $v_{t_{i}} \rightarrow v$ as $i \rightarrow \infty$ the assertion follows by the definition of $D V_{0}$.

Based on $V_{0}$ and using the techniques from [4] we can now construct the stabilizing feedback law for system (3.4). To this end for $\beta>0$ we consider the approximation of $V_{0}$ via the inf-convolution

$$
V_{\beta}(x)=\inf _{y \in \mathbb{R}^{n}}\left\{V_{0}(y)+\frac{\|x-y\|^{2}}{2 \beta^{2}}\right\} .
$$

Observe that $V_{\beta}$ is locally Lipschitz and $V_{\beta} \rightarrow V_{0}$ as $\beta \rightarrow 0$.

Proposition 4.2. For each $\mu \in(0, \rho)$ there exists $\beta>0$ such that the function $V_{\beta}$ is a Lipschitz continuous clf which is homogeneous with degree 1 (with respect to the standard dilation) and satisfies

$$
\min _{v \in \operatorname{cof}(x, U)} D V_{\beta}(x ; v) \leq-2 \mu V_{\beta}(x) .
$$

Furthermore there exists a feedback law $F: \mathbb{R}^{n} \rightarrow U$ satisfying $F(\alpha x)=F(x)$ for all $x \in \mathbb{R}^{n}, \alpha>0$ and constants $h>0$ and $C>0$ such that any $\pi$-trajectory corresponding to some partition $\pi$ with $d(\pi) \leq h$ satisfies

$$
\left\|x_{\pi}\left(t, x_{0}, F\right)\right\| \leq C e^{-\mu}\left\|x_{0}\right\| .
$$

Proof. By its definition $V_{\beta}$ is obviously positive definite. Now for each $x \in \mathbb{R}^{n}$ we denote by $y_{\beta}(x)$ a point realizing the minimum on the right hand side of (4.1). Since $V_{0}$ is homogeneous with degree 1 we have that

$$
\left\{V_{0}(\alpha y)+\frac{\|\alpha x-\alpha y\|^{2}}{2 \beta^{2}}\right\}=\alpha^{2}\left\{V_{0}(y)+\frac{\|x-y\|^{2}}{2 \beta^{2}}\right\}
$$

and thus in particular $V_{\beta}$ is also homogeneous with degree 1 , hence proper, and we can choose $y_{\beta}(x)$ in such a way that $y_{\beta}(\alpha x)=\alpha y_{\beta}(x)$. Since $V_{0}$ is strictly increasing along the rays $\alpha x$ in $\alpha>0$ it follows that $\left\|y_{\beta}(x)\right\| \leq\|x\|$.

Now we define

$$
\zeta_{\beta}(x):=\frac{x-y_{\beta}(x)}{2 \beta^{2}}
$$

which implies $\zeta_{\beta}(\alpha x)=\alpha \zeta_{\beta}(x)$. 
By [4, Lemma III.1 and III.2] (or by straightforward calculations) for this vector we can deduce the inequalities

$$
V_{\beta}(x+\tau v) \leq V_{\beta}(x)+\tau\left\langle\zeta_{\beta}(x), v\right\rangle+\frac{\tau^{2}\|v\|^{2}}{2 \beta^{2}}
$$

and

$$
V_{0}\left(y_{\beta}(x)+\tau v\right) \geq V_{0}\left(y_{\beta}(x)\right)+\tau\left\langle\zeta_{\beta}(x), v\right\rangle-\frac{\tau^{2}\|v\|^{2}}{2 \beta^{2}},
$$

i.e. $\zeta_{\beta}(x)$ is a proximal supergradient of $V_{\beta}$ in $x$ and a proximal subgradient of $V_{0}$ in $y_{\beta}(x)$ (see e.g. [3] for an exposition of these concepts). We choose the feedback $F(x)$ in such a way that

$$
\left\langle\zeta_{\beta}(x), f(x, F(x))\right\rangle=\inf _{u \in U}\left\langle\zeta_{\beta}(x), f(x, u)\right\rangle
$$

and $F(\alpha x)=F(x)$ for all $x \in \mathbb{R}^{n} \backslash\{0\}$ and all $\alpha>0$. The value $F(0)$ can be chosen arbitrary.

Now consider points $x \in \mathbb{R}^{n}$ with $\|x\|=1$, i.e. $x \in \mathbb{S}^{n-1}$. For these points the Hölder continuity of $V_{0}$ (which is inherited from the Hölder continuity of $v_{\delta}$ ) and the definitions of $V_{\beta}$ and $\zeta_{\beta}$ imply

$$
\frac{1}{2 \beta^{2}}\left\|y_{\beta}(x)-x\right\|^{2} \leq V_{0}(x)-V_{0}\left(y_{\beta}(x)\right) \leq H\left\|y_{\alpha}(x)-x\right\|^{\nu}
$$

and thus

$$
\left\|\zeta_{\beta}(x)\right\|\left\|y_{\beta}(x)-x\right\| \leq H \beta^{\frac{2 \nu}{2-\nu}}
$$

where $H>0$ and $\nu \in(0,1]$ denote the Hölder constant and exponent of $V_{0}$ on $\left\{x \in \mathbb{R}^{n} \mid\|x\| \leq 1\right\}$. From (4.5) and the definition of $V_{\beta}$ we immediately obtain

$$
\left|V_{0}\left(y_{\beta}(x)\right)-V_{\beta}(x)\right| \leq H \beta^{\frac{2 \nu}{2-\nu}}
$$

Now the Lipschitz continuity of $f$ implies that

$$
\left\langle\zeta_{\beta}(x), f(x, F(x))\right\rangle \leq \min _{u \in U}\left\langle\zeta_{\beta}(x), f\left(y_{\beta}(x), u\right)\right\rangle+L\left\|\zeta_{\beta}(x) \mid\right\|\left\|y_{\beta}(x)-x\right\|
$$

and by (4.4) and the definition of $D V_{0}$ it follows that $\left\langle\zeta_{\beta}(x), v\right\rangle \leq D V_{0}\left(y_{\beta}(x), v\right)$ for all $v \in \mathbb{R}^{n}$. Thus by the linearity of the scalar product and Proposition 4.1 we can conclude

$$
\min _{u \in U}\left\langle\zeta_{\beta}(x), f\left(y_{\beta}(x), u\right)\right\rangle=\min _{v \in \operatorname{cof}\left(y_{\beta}(x), U\right)}\left\langle\zeta_{\beta}(x), v\right\rangle \leq-2 \rho V_{0}\left(y_{\beta}(x)\right) .
$$

Combining these inequalities with (4.5) and (4.6) yields

$$
\left\langle\zeta_{\beta}(x), f(x, F(x))\right\rangle \leq-2 \rho V_{\beta}(x)+2 \rho H \beta^{\frac{2 \nu}{2-\nu}}+L H \beta^{\frac{2 \nu}{2-\nu}} .
$$

Defining

$$
f_{x}^{\tau}:=\frac{1}{\tau} \int_{0}^{\tau} f(x(t, x, F(x)), F(x)) d t
$$


and using $M:=\sup _{\|x\| \leq 2, u \in U} f(x, u)$ and the Lipschitz continuity of $f$ for $\tau>0$ sufficiently small we obtain

$$
\left\|f_{x}^{\tau}-f(x, F(x))\right\| \leq M L \tau, \quad\left\|f_{x}^{\tau}\right\| \leq M .
$$

Thus by (4.3), (4.7), and the fact that $\left\|\zeta_{\beta}(x)\right\| \leq C_{\beta}$ for all $x \in \mathbb{S}^{n-1}$ and some suitable $C_{\beta}>0$ we can conclude

$$
\begin{aligned}
V_{\beta}(x(\tau, x, F(x)))-V_{\beta}(x) & =V_{\beta}\left(x+\tau f_{x}^{\tau}\right)-V_{\beta}(x) \\
& \left.\leq \tau\left\langle\zeta_{\beta}(x), f_{x}^{\tau}\right)\right\rangle+\frac{\tau^{2}\left\|f_{x}^{\tau}\right\|^{2}}{2 \beta^{2}} \\
& \leq \tau\left\langle\zeta_{\beta}(x), f(x, F(x))\right\rangle+M L \tau^{2}\left\|\zeta_{\beta}(x)\right\|+\frac{\tau^{2} M^{2}}{2 \beta^{2}} \\
& \leq \tau\left(-2 \rho V_{\beta}(x)+(2 \rho+L) H \beta^{\frac{2 \nu}{2-\nu}}\right)+\tau^{2}\left(M L C_{\beta}+\frac{M^{2}}{2 \beta^{2}}\right)
\end{aligned}
$$

Denoting

$$
\gamma_{\beta}:=\sup _{x \in \mathbb{S}^{n-1}} \frac{(2 \rho+L) H \beta^{\frac{2 \nu}{2-\nu}}}{V_{\beta}(x)}, \quad \tilde{C}_{\beta}:=\sup _{x \in \mathbb{S}^{n-1}} \frac{M L C_{\beta}}{V_{\beta}(x)}+\frac{M^{2}}{2 \beta^{2} V_{\beta}(x)},
$$

and exploiting homogenity of $x(\cdot, x, F(x))$ and $V_{\beta}$ we obtain for arbitrary $x \neq 0$

$$
V_{\beta}(x(\tau, x, F(x)))-V_{\beta}(x) \leq \tau\left(-2 \rho+\gamma_{\beta}\right) V_{\beta}(x)+\tau^{2} \tilde{C}_{\beta} V_{\beta}(x)
$$

which immediately implies both assertions since $\gamma_{\beta} \rightarrow 0$ as $\beta \rightarrow 0$.

This proposition shows the stabilization for systems of type (3.4). The following theorem shows how this result can be translated to the general homogeneous-in-thestate system from Definition 3.1.

Theorem 4.3. Consider system (3.1) satisfying Definition 3.1 with dilation matrix $\Lambda_{\alpha}$, minimal power $k>0$, and degree $\tau \in(-k, \infty)$, and assume asymptotic controllability. Then there exists $\mu>0$ and a clf $V$ being Lipschitz on $\mathbb{R}^{n} \backslash\{0\}$, satisfying

$$
V\left(\Lambda_{\alpha}(x)\right)=\alpha^{2 k} V(x)
$$

and

$$
\min _{v \in \operatorname{cof}(x, U)} D V_{\beta}(x ; v) \leq-2 \mu N^{\tau}(x) V_{\beta}(x)
$$

for the function $N$ from (2.3).

Furthermore there exists a feedback law $F: \mathbb{R}^{n} \rightarrow U$ satisfying $F\left(\Lambda_{\alpha} x\right)=F(x)$ for all $x \in \mathbb{R}^{n}, \alpha>0$ such that the corresponding sampled closed loop system is either

(i) semi-globally stable (if $\tau>0$ ), or

(ii) globally uniformly exponentially stable (if $\tau=0$ ), or

(iii) globally practically exponentially stable (if $\tau<0$ ) with fixed sampling rate.

Proof. Obviously if the system defined by $f$ is asymptotically controllable, then the transformed system defined by $\bar{f}$ is asymptotically controllable. Thus from Proposition 4.2 we obtain $\bar{V}=V_{\beta}$ and $\bar{F}=F$ satisfying the assertion for $\bar{f}$ which is homogeneous-in-the-state with $\Lambda_{\alpha}=\alpha \mathrm{Id}, k=1$ and $\tau=0$. 
We start by showing the result for the system defined by $\tilde{f}(x, u)=\bar{f}(x, u)\|x\|^{\gamma}$ being homogeneous-in-the-state with with $\Lambda_{\alpha}=\alpha \mathrm{Id}, k=1$ and $\tau=\gamma$. Let $\tilde{V}(x)=$ $\bar{V}(x)$. Then we immediately obtain

$$
\min _{v \in \operatorname{co} \tilde{f}(x, U)} D \tilde{V}(x ; v)=\|x\|^{\gamma} \min _{v \in \operatorname{co} \bar{f}(x, U)} D \tilde{V}(x ; v) \leq-\|x\|^{\gamma} 2 \mu \tilde{V}(x) .
$$

Now observe that for each control function $u(\cdot) \in \mathcal{U}$ the trajectories $\tilde{x}$ and $\bar{x}$ of these systems satisfy

$$
\tilde{x}\left(t, x_{0}, u(\cdot)\right)=\bar{x}\left(\bar{t}(t), x_{0}, u(\tilde{t}(\cdot))\right)
$$

where $\tilde{t}(t)$ denotes the inverse of $\bar{t}(t)$ which is defined by

$$
\left.\bar{t}(t)=\int_{0}^{t} \| \tilde{x}\left(\tau, x_{0}, u(\cdot)\right)\right) \|^{\gamma} d t
$$

and thus is well defined as long as the solution $\tilde{x}\left(t, x_{0}, u(\cdot)\right)$ exists. If both $\tilde{x}$ and $\bar{x}$ uniquely exist for all $t \geq 0$ it is immediate that $\bar{t}(t) \rightarrow \infty$ as $t \rightarrow \infty$.

Setting $\tilde{F}(x)=\bar{F}(x)$ a $\tilde{\pi}$-trajectory $\tilde{x}_{\tilde{\pi}}\left(t, x_{0}, \tilde{F}\right)$ of

$$
\dot{\tilde{x}}=\tilde{f}(\tilde{x}, \tilde{F}(\tilde{x}))
$$

on some interval $[0, T]$ on which $\tilde{x}_{\pi}$ exists becomes a $\bar{\pi}$-trajectory $\bar{x}_{\bar{\pi}}\left(\bar{t}(t), x_{0}, \bar{F}\right)$ of

$$
\dot{\bar{x}}=\bar{f}(\bar{x}, \bar{F}(\bar{x}))
$$

where $\bar{\pi}=\left(\bar{t}_{i}\right)_{i \in \mathbb{N}_{0}}$ is given by $\bar{t}_{i}=\bar{t}\left(\tilde{t}_{i}\right)$ with $\tilde{\pi}=\left(\tilde{t}_{i}\right)_{i \in \mathbb{N}_{0}}$. Now we distinguish the three cases:

(i) $\gamma>0$ : By the choice of $\bar{F}$ there exist $C, \sigma, h>0$ such that inequality (4.2) holds for each $\bar{\pi}$-trajectory $\bar{x}_{\bar{\pi}}$ of $(4.10)$ with $d(\bar{\pi}) \leq h$ and each $x \in \mathbb{R}^{n}$. Now consider a compact set $K \subset \mathbb{R}^{n}$ with $0 \in \operatorname{int} K$. Let $C_{K}:=\sup _{x \in K}\|x\|$, consider a $\tilde{\pi}$-trajectory $\tilde{x}_{\tilde{\pi}}\left(t, x_{0}, \tilde{F}\right)$ of $(4.9)$ with $d(\tilde{\pi}) \leq h\left(C C_{K}\right)^{-\gamma}$ and $x \in K$, and assume that there exists a (minimal) time $t^{*}>0$ such that $\left\|\tilde{x}_{\tilde{\pi}}\left(t^{*}, x_{0}, \tilde{F}\right)\right\|=C\|x\|$. W.l.o.g. we may assume $t^{*}=\tilde{t}_{l} \in \tilde{\pi}$ for some $l>0$, otherwise we may reduce the sampling interval containing $t^{*}$. Then since $\left\|\tilde{x}_{\tilde{\pi}}\left(t, x_{0}, \tilde{F}\right)\right\| \leq C C_{K}$ for all $t \in\left[0, \tilde{t}_{l}\right]$ the rescaled $\bar{\pi}$ satisfies $\bar{t}_{i}-\bar{t}_{i-1} \leq h$ for all $i=1, \ldots, l$, thus we obtain

$$
\left\|\tilde{x}_{\tilde{\pi}}\left(\tilde{t}_{l}, x_{0}, \tilde{F}\right)\right\|=\left\|\bar{x}_{\bar{\pi}}\left(\bar{t}_{l}, x_{0}, \bar{F}\right)\right\| \leq C e^{-\mu \bar{t}_{l}}\left\|x_{0}\right\|<C\left\|x_{0}\right\|
$$

contradicting the choice of $t^{*}=\tilde{t}_{l}$. Thus $\left\|\tilde{x}_{\tilde{\pi}}\left(t, x_{0}, \tilde{F}\right)\right\| \leq C\|x\|$ holds for all $t \geq 0$, and hence $d(\bar{\pi}) \leq h$, implying

$$
\left\|\tilde{x}_{\tilde{\pi}}\left(t, x_{0}, \tilde{F}\right)\right\| \leq C e^{-\mu \bar{t}(t)}\left\|x_{0}\right\|
$$

which implies the desired stability estimate with $\beta(\|x\|, t)=C e^{-\mu \tilde{t}(t)}\|x\|$ which is of class $\mathcal{K} \mathcal{L}$ because the corresponding trajectories stay inside some compact set, thus exist for all $t \geq 0$, and are unique since $\gamma>0$, hence $\bar{t}(t) \rightarrow \infty$ as $t \rightarrow \infty$.

(ii) $\gamma=0$ : In this case the assumption follows immediately from Proposition 4.2.

(iii) $\gamma<0$ : As in case (i) there exist $C, \sigma, h>0$ such that inequality (4.2) holds for each $\bar{\pi}$-trajectory $\bar{x}_{\bar{\pi}}$ of (4.10) with $d(\bar{\pi}) \leq h$ and each $x \in \mathbb{R}^{n}$. Consider a compact set $K \subset \mathbb{R}^{n}$ and an open set $B \subset \mathbb{R}^{n}$ with $0 \in B \subset K$. Let $C_{K}=\sup _{x \in K}\|x\|, C_{B}=$ $\inf _{x \notin B}\|x\| / 2>0$. By continuous dependence on the initial value and compactness we 
can choose $s>0$ such that $\left\|\tilde{x}\left(t, x_{0}, u\right)\right\| \leq 2 C^{-1} C_{B}$ for all $\left\|x_{0}\right\|=C^{-1} C_{B}$, all $u \in U$, and all $t \in[0, s]$. Then (3.3) implies (recall $\tau=\gamma<0$ and $\Lambda_{\alpha}=\alpha \mathrm{Id}$ ) the inequality $\left\|\tilde{x}\left(t, x_{0}, u\right)\right\| \leq 2\left\|x_{0}\right\|$ for all $\left\|x_{0}\right\| \geq C^{-1} C_{B}$, all $u \in U$ and all $t \in[0, s]$.

Now pick an arbitrary $\tilde{\pi}$-trajectory $\tilde{x}_{\tilde{\pi}}\left(t, x_{0}, \tilde{F}\right)$ of $(4.9)$ with $\tilde{\pi}$ satisfying $d(\tilde{\pi}) \leq$ $\min \left\{h\left(C^{-1} C_{B}\right)^{-\gamma}, s\right\}$ and $x_{0} \in K,\left\|x_{0}\right\| \geq C^{-1} C_{B}$, and consider an interval $\left[t_{*}, t^{*}\right]$ such that $\left\|\tilde{x}_{\tilde{\pi}}(t, x, \tilde{F})\right\| \geq C^{-1} C_{B}$ for all $t \in\left[t_{*}, t^{*}\right]$. Then we either have $t_{*}=0=\tilde{t}_{0}$ or there exist sampling times such that $t_{*} \in\left[\tilde{t}_{i-1}, \tilde{t}_{i}\right]$. In this case by $d(\tilde{\pi}) \leq s$ and the choice of $s$ we obtain $\left\|\tilde{x}_{\tilde{\pi}}\left(\tilde{t}_{i}, x_{0}, \tilde{F}\right)\right\| \leq 2\left\|\tilde{x}_{\tilde{\pi}}\left(t_{*}, x_{0}, \tilde{F}\right)\right\|$.

Analogously to the case $\gamma>0$ the choice of $d(\tilde{\pi})$ now implies $d(\bar{\pi}) \leq h$ and thus

$$
\left\|\tilde{x}_{\tilde{\pi}}\left(t, x_{0}, \tilde{F}\right)\right\| \leq C e^{-\mu\left(\bar{t}(t)-\bar{t}\left(\tilde{t}_{i}\right)\right)}\left\|\tilde{x}_{\tilde{\pi}}\left(\tilde{t}_{i}, x_{0}, \tilde{F}\right)\right\|
$$

for all $t \in\left[\tilde{t}_{i}, t^{*}\right]$. This estimate implies that for each trajectory there exists a (minimal) time $T \geq 0$ such that the trajectory hits the set $\left\{x \in \mathbb{R}^{n}\|\| x \| \leq C^{-1} C_{B}\right\}$, and up to that time (4.11) implies the desired estimate with $\beta\left(\left\|x_{0}\right\|, t\right)=C e^{-\mu \bar{t}(t)}\|x\| \leq$ $C e^{-\mu\left(C C_{K}\right)^{\gamma} t}\left\|x_{0}\right\|$. After that time $T$, whenever the trajectory leaves this set at some time $t_{*}>T$ inequality (4.11) implies that it will enter again at some time $t^{*}>t_{*}$ and satisfies $\left\|\tilde{x}_{\tilde{\pi}}\left(t, x_{0}, \tilde{F}\right)\right\| \leq \tilde{C} C_{B} / 2 \leq C_{B}$ for all $t \in\left[t_{*}, t^{*}\right]$. Hence $\left\|\tilde{x}_{\tilde{\pi}}\left(t, x_{0}, \tilde{F}\right)\right\| \leq C_{B}$ for all $t \geq T$, and since $\|x\| \leq C_{B}$ implies $x \in B$ we obtain the practical stability property.

So far we have shown the existence of $\tilde{V}$ and $\tilde{F}$ satisfying the assumptions of the theorem for $\tilde{f}$, hence it remains to translate the results to $f$. To this end we define $V(x)=\tilde{V}(\Psi(x))$ and $F(x)=\tilde{F}(\Psi(x))$. This implies

$$
D V(x ; f(x, u))=D \tilde{V}(\Psi(x) ; \tilde{f}(\Psi(x), u))
$$

and

$$
x_{\pi}(t, x, F)=\tilde{x}_{\pi}(t, \Psi(x), \tilde{F})
$$

and thus immediately the assertion since $\|\Psi(x)\|=N^{k}(x)$.

5. Numerical approximation of $V$ and $F$. In this section we briefly explain how a numerical approximation to the clf $V$ and the feedback law $F$ can be computed.

Unfortunately, up to now no numerical method for the approximation of $v_{\delta}, V_{0}$ or $V_{\beta}$ is known, which also gives an approximation of the super- or subgradients and thus allows the approximation of $F$. However, if we slightly change our feedback concept (or, more precisely, the notion of a closed loop system) an approximation is possible. For this purpose we introduce the following definition.

Definition 5.1. Let $h: \mathbb{R}^{n} \rightarrow \mathbb{R}^{+}$be an arbitrary map. A feedback law $F^{h}:$ $\mathbb{R}^{n} \rightarrow U$ is called a discrete feedback, if we apply it as a sampled feedback according to Definition 2.3 with fixed intersampling times $\Delta t_{i}=h\left(x_{\pi}\left(t_{i}, x_{0}, F^{h}\right)\right)$.

This definition generalizes the one given in [7] in the sense that the time step $h$ now may depend on $x$. The name "discrete feedback" is motivated by the fact that the resulting system can be written as a discrete time system $x_{i+1}=x\left(h\left(x_{i}\right), x_{i}, F^{h}\left(x_{i}\right)\right)$ for which $F^{h}$ is a feedback law in the classical sense.

Returning to our simplified system (3.4), and again fixing some $\rho>0$ and $\delta \in$ $(0, \rho)$ we can apply the results from $[7,8]$, observing that the structural assumptions on the system in these references (i.e. bi- or semilinearity, accessibility, convexity of $U$ ) are only needed in order to show $v_{\delta}(x)<0$. In particular all the numerical approximation results remain valid, thus we can proceed as in $[7,8]$ and (i) approximate $\mathcal{U}$ by 
piecewise constant control functions, (ii) approximate the trajectories and the integral by numerical schemes, and (iii) compute an approximation of $v_{\delta}$ on a grid discretizing the state space $\mathbb{S}^{n-1}$. Proceeding this way for any given $\varepsilon>0$ we find $h>0$ and numerically computable functions $v_{\delta}^{h}: \mathbb{S}^{n-1} \rightarrow \mathbb{R}$ and $F_{\mathbb{S}}^{h}: \mathbb{S}^{n-1} \rightarrow U$ such that

$$
v_{\delta}^{h}(s) \leq-\rho+\varepsilon
$$

and

$$
v_{\delta}^{h}(s)+\varepsilon \geq \int_{0}^{h} q\left(s\left(t, s, F_{\mathbb{S}}^{h}(s)\right), F_{\mathbb{S}}^{h}(s)\right) d t+e^{-\delta h} v_{\delta}^{h}\left(s\left(h, s, F_{\mathbb{S}}^{h}(s)\right)\right)
$$

hold for each $s \in \mathbb{S}^{n-1}$. This function $v_{\delta}^{h}$ is the function $\tilde{v}_{\delta}^{a}$ from [7]; Inequality (5.1) follows from [7, Theorems 3.3, 5.3, and Inequality (5.4)], Inequality (5.2) is easily extracted from the proofs of [7, Lemma 5.1, Lemma 5.2, and Proposition 5.4] using again [7, Inequality (5.4)]. The feedback $F_{\mathbb{S}}^{h}$ is defined by choosing a control value minimizing the right hand side of (5.2) using the numerical approximations of the trajectory and the integral. Observe that the state space $\mathbb{S}^{n-1}$ to be discretized here is somewhat more difficult to handle that the projective space $\mathbb{P}^{n-1}$ appearing in [7,8], since for $n \geq 3$ a single map cannot be sufficient for the parametrization of $\mathbb{S}^{n-1}$ without introducing singularities. Hence numerically one either needs to work directly on $\mathbb{S}^{n-1}$, or one has to compute the solution using two parametrizations (e.g. the stereographic projection from the north and south pole), and consequently two grids for the representation of $v_{\delta}^{h}$ in local coordinates. This method was used for the second example in Section 7.

Defining $F^{h}(x)=F_{\mathbb{S}}^{h}(x /\|x\|)$ analogous to Proposition 4.1 we can conclude that the function

$$
V^{h}(x):=e^{2 v_{\delta}^{h}(x /\|x\|)}\|x\|
$$

is homogeneous, proper, positive definite, and satisfies

$$
V^{h}\left(x\left(h, x, F^{h}(x)\right)\right) \leq\left(1-2 h \rho+M\left(\varepsilon+\varepsilon h+h^{2}\right)\right) V^{h}(x)
$$

for some suitable constant $M>0$ independent of $h$ and $\varepsilon$, i.e. for any $\rho^{\prime} \in(0, \rho)$ there exist sufficiently small $h>0$ and $\varepsilon>0$ such that

$$
V^{h}\left(x\left(h, x, F^{h}(x)\right)\right) \leq\left(1-2 h \rho^{\prime}\right) V^{h}(x) .
$$

Thus the function $V^{h}$ is a (discrete time) Lyapunov function for the system controlled by the discrete feedback $F^{h}$ according to Definition 5.1 with $h(x) \equiv h$, which immediately implies (exponential) stability.

As in Theorem 4.3 we can retranslate this result to arbitrary homogeneous-in-thestate systems. Analogous to the proof of this theorem denote the functions obtained for (3.4) by $\bar{F}^{h}, \bar{V}^{h}$, and $\bar{h}$. For the retranslation from $\bar{f}$ to $\tilde{f}$ we can use $\tilde{F}^{h}=\bar{F}^{h}$, and $\tilde{F}^{h}=\bar{V}^{h}$, however, following (4.8) we now have to use $\tilde{h}(x)=\tilde{t}(\bar{h}, x)$ as intersampling times, where

$$
\tilde{t}(\bar{h}, x)=\int_{0}^{\bar{h}}\left\|\bar{x}\left(\tau, x, F^{h}(x)\right)\right\|^{-\gamma} d \tau .
$$

Passing from $\tilde{f}$ to $f$ we define-again analogously to the Proof of Theorem 4.3the feedback $F^{h}(x)=\tilde{F}^{h}(\Psi(x))$, the Lyapunov function $V^{h}(x)=\tilde{V}^{h}(\Psi(x))$ and 
the intersampling time $h(x)=\tilde{h}(\Psi(x))$. This way it is straightforward to see that (5.3) - now for the $x$-dependent $h$-remains valid and thus stability follows.

Observe that the time steps $h(x)$ are bounded from below by some positive constant on each compact set iff $\tau \geq 0$, and they are bounded on each open set not containing the origin iff $\tau \leq 0$. In this way they behave just like the sampling rate for the theoretical feedback law from Theorem 4.3, however, here the stability is only guaranteed for these fixed intersampling times $h(x)$ and not for smaller ones as allowed in Definition 2.5.

6. Stabilization of homogeneous systems. We now return to the homogeneous system from Definition 2.1. The idea of the proof of Theorem 2.6 lies in the fact, that for any asymptotically controllable homogeneous system we can find an asymptotically controllable homogeneous-in-the-state system. For this we find a clf and a stabilizing feedback law by Theorem 4.3, which-retranslated to the homogeneous system - have the properties as stated in Theorem 2.6.

We start by constructing the desired system: Given some homogeneous system satisfying

$$
g\left(\Lambda_{\alpha} x, \Delta_{\alpha} u\right)=\alpha^{\tau} \Lambda_{\alpha}(x, u)
$$

we define

$$
f(x, u):=g\left(x, \Delta_{N(x)} u\right) .
$$

Then it is immediate from the property $N\left(\Lambda_{\alpha} x\right)=\alpha N(x)$ of the dilated norm $N$ that

$$
\begin{aligned}
f\left(\Lambda_{\alpha} x, u\right) & =g\left(\Lambda_{\alpha} x, \Delta_{N\left(\Lambda_{\alpha} x\right)} u\right)=g\left(\Lambda_{\alpha} x, \Delta_{\alpha N(x)} u\right) \\
& =g\left(\Lambda_{\alpha} x, \Delta_{\alpha} \Delta_{N(x)} u\right)=\alpha^{\tau} \Lambda_{\alpha} g\left(x, \Delta_{N(x)} u\right) \\
& =\alpha^{\tau} \Lambda_{\alpha} f(x, u)
\end{aligned}
$$

i.e. $f$ is homogeneous-in-the-state.

The following proposition gives the asymptotic controllability.

Proposition 6.1. Consider a system (2.1) satisfying Definition 2.1. Assume that the system is asymptotically controllable. Then there exists a compact set of controlvalues $U \subset W$ such that the homogeneous-in-the-state system (6.1) is asymptotically controllable using control functions with values in $U$.

Proof. First observe that due to (3.3) it is sufficient to show that there exists a compact $U \subset W$ and a time $T>0$ such that any initial value $x_{0}$ with $N\left(x_{0}\right)=1$ can be steered to some point $x_{1}$ with $N\left(x_{1}\right) \leq 1 / 2$ in some time $t<T$ using a measurable control $u(\cdot)$ with $u(t) \in U$ for almost all $t \geq 0$. With this property asymptotic controllability easily follows by induction from (3.3).

In order to show the existence of this $U$ first observe that, denoting the trajectories of $f$ and $g$ by $x_{f}$ and $x_{g}$, respectively, the equality

$$
x_{f}\left(t, x_{0}, u(\cdot)\right)=x_{g}\left(t, x_{0}, w(\cdot)\right), \quad \text { with } u(t)=\Delta_{N\left(x_{g}\left(t, x_{0}, w(\cdot)\right)\right)}^{-1} w(t)
$$

holds. Now consider the initial values $x_{0} \in N^{-1}(1)$. For each of these points there exists a control $w_{x_{0}}(\cdot) \in \mathcal{W}$ such that $N\left(x_{g}\left(t_{x_{0}}, x_{0}, w_{x_{0}}(\cdot)\right)\right)=1 / 3$. Now by continuous dependence of the solution on the initial value we obtain that for each $x_{0}$ there exists an open neighbourhood $B_{x_{0}} \ni x_{0}$ such that

$$
N\left(x_{g}\left(t_{x_{0}}, x, w_{x_{0}}(\cdot)\right)\right) \leq 1 / 2
$$


for all $x \in B_{x_{0}}$. Since $N^{-1}(1)$ is compact and is covered by the $B_{x_{0}}$ we find a finite number $M \in \mathbb{N}$ of points $x_{0}^{i}, i=1, \ldots, M$, such that the sets $U_{x_{0}^{i}}, i=1, \ldots, M$ cover $N^{-1}(1)$. Thus setting $t_{i}=t_{x_{0}^{i}}$ and $w_{i}(\cdot)=w_{x_{0}^{i}}(\cdot)$ for each $x_{0} \in N^{-1}(1)$ there exists a number $i \in\{1, \ldots, M\}$ such that

$$
N\left(x_{g}\left(t_{i}, x_{0}, w_{i}(\cdot)\right)\right) \leq 1 / 2 .
$$

Now we choose $u_{i}(t)=\Delta_{N\left(x_{g}\left(t, x_{0}, w_{i}(\cdot)\right)\right)}^{-1} w_{i}(t)$ for all $t \in\left[0, t_{i}\right], u_{i}(t) \in W$ arbitrary for $t>t_{i}$. Then by (6.2) we immediately obtain

$$
N\left(x_{f}\left(t_{i}, x, u_{i}(\cdot)\right)\right) \leq 1 / 2
$$

for each $x_{0} \in N^{-1}(1)$ and some suitable $i \in\{1, \ldots, M\}$. Since the functions $w_{i}(\cdot)$ are locally essentially bounded, i.e. essentially bounded on $\left[0, t_{i}\right]$, we can conclude that the functions $u_{i}(\cdot)$ are essentially bounded. Thus $\left\|u_{i}(\cdot)\right\|_{\infty}$ is finite for each $i=1, \ldots, M$ and also $\sup _{i=1, \ldots, M}\left\|u_{i}(\cdot)\right\|_{\infty}$ is finite, hence there exists a compact $U \subset W$ such that $u_{i}(t) \in U$ for almost all $t>0$ and all $i=1, \ldots, M$.

Now we can turn to the proof of Theorem 2.6.

Proof of Theorem 2.6. Consider the system homogeneous-in-the-state as defined by (6.1) with $U \subset W$ from Proposition 6.1. For this system from Theorem 4.3 we obtain a clf $V_{f}$ and a feedback $F_{f}$. Setting $V=V_{f}$ and $F(x)=\Delta_{N(x)} F_{f}(x)$ we immediately obtain the assertion.

7. Examples. Let us, finally, illustrate our results by two examples. The first example

$$
g(x, w)=\left(\begin{array}{l}
x_{1}+w \\
3 x_{2}+x_{1} w^{2}
\end{array}\right)
$$

for $x=\left(x_{1}, x_{2}\right)^{T} \in \mathbb{R}^{2}, w \in W=\mathbb{R}$, is taken from [22] where it has been shown that a stabilizing continuous and homogeneous feedback law cannot exist for this system. The vector field $g$ is homogeneous with $\Lambda_{\alpha}=\operatorname{diag}\left(\alpha, \alpha^{3}\right)$ and $\Delta_{\alpha}=\alpha$. Thus we obtain $N(x)=\left(x_{1}^{6}+x_{2}^{2}\right)^{1 / 6}$. For system (7.1) a stabilizing discrete feedback $F^{h}$ has been computed numerically using the techniques of Section 5. Analyzing the switching curves of the numerical feedback in this case it was easy to derive the feedback

$$
F(x)=\left\{\begin{array}{rr}
N(x), & x_{1} \leq-x_{2}^{3} \\
-N(x), & x_{1}>-x_{2}^{3}
\end{array}\right.
$$

stabilizing the sampled system for all sufficiently small sampling rates. Figure 7.1 shows the corresponding (numerically simulated) sampled trajectories for some initial values, here the intersampling times have been chosen as $\Delta t_{i}=0.01$ for all $i \in \mathbb{N}_{0}$.

The second example is the nonholonomic integrator given by Brockett [2] as an example for a system being asymptotically null controllable but not stabilizable by a continuous feedback law. In suitable coordinates (cf. [24], where also the physical meaning is discussed) it reads

$$
g(x, w)=\left(\begin{array}{r}
w_{1} \\
w_{2} \\
x_{1} w_{2}
\end{array}\right)
$$

for $x=\left(x_{1}, x_{2}, x_{3}\right)^{T} \in \mathbb{R}^{3}, w=\left(w_{1}, w_{2}\right)^{T} \in W=\mathbb{R}^{2}$. For this $g$ we obtain homogenity with $\Lambda_{\alpha}=\operatorname{diag}\left(\alpha, \alpha, \alpha^{2}\right)$ and $\Delta_{\alpha}=\operatorname{diag}(\alpha, \alpha)$, hence $N(x)=\left(x_{1}^{4}+x_{2}^{4}+x_{3}^{2}\right)^{1 / 4}$. 


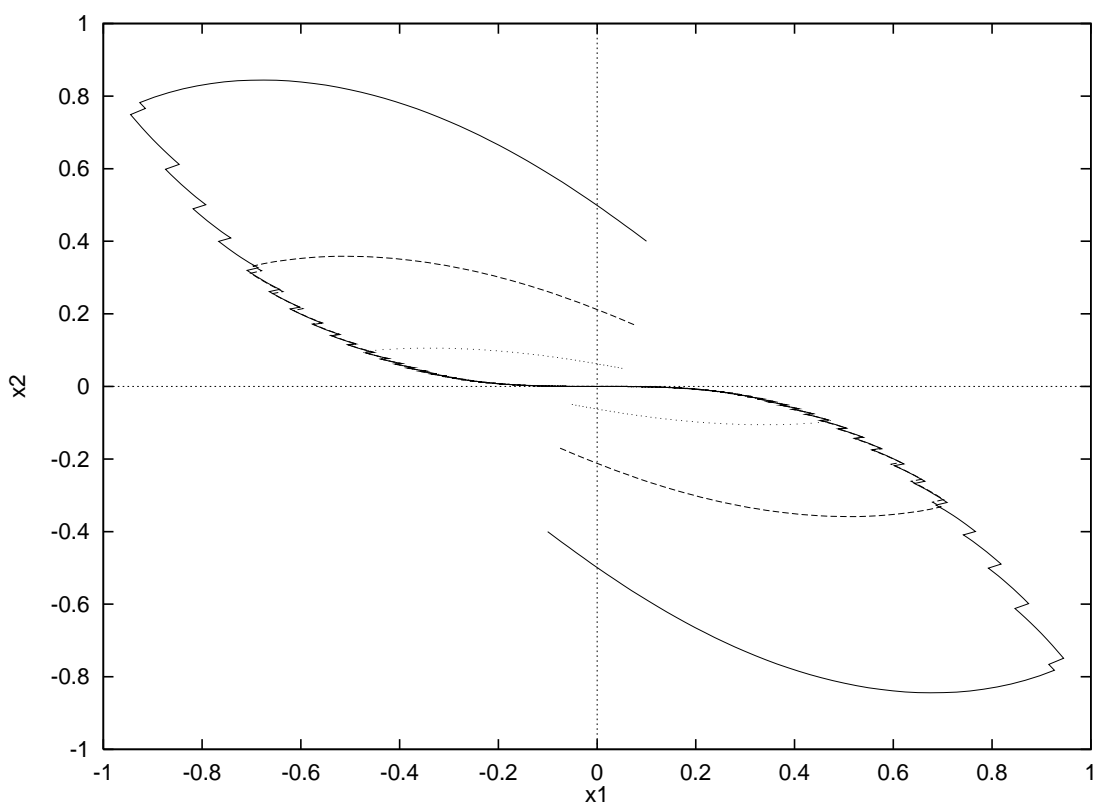

FIG. 7.1. Trajectories for stabilized system (7.1)

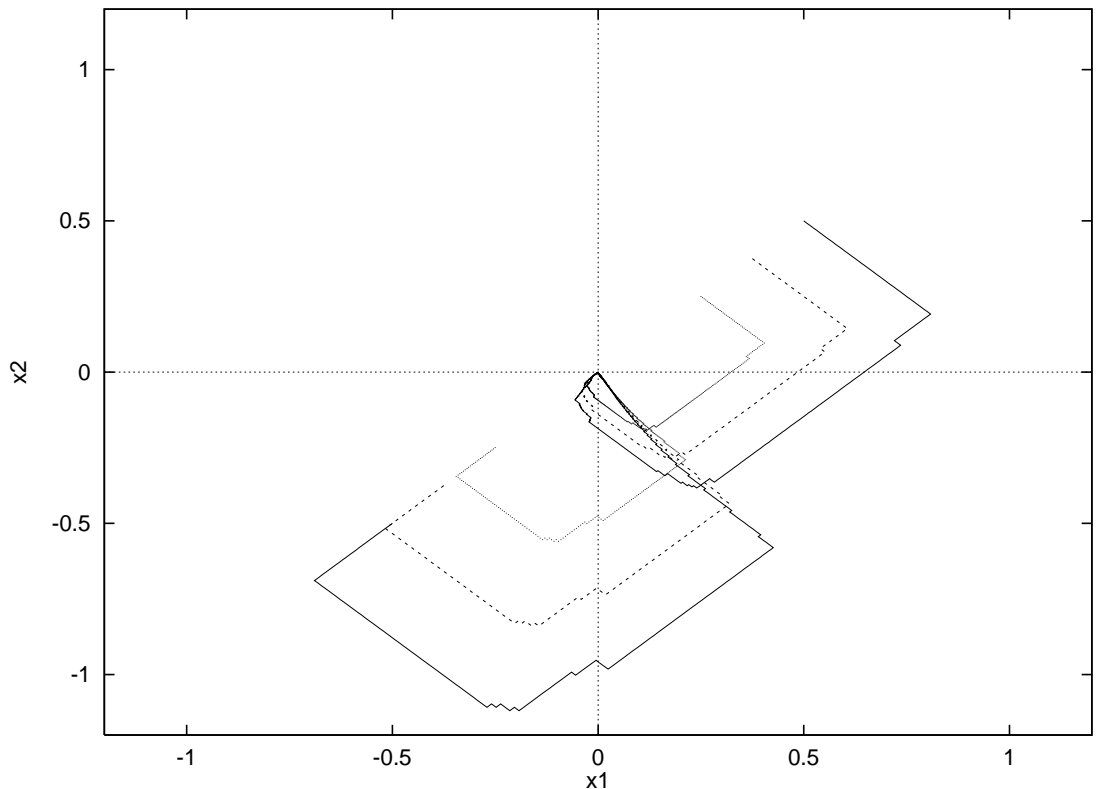

FIG. 7.2. Trajectories for stabilized system (7.2), projected to the $\left(x_{1}, x_{2}\right)$ plane

Again a stabilizing discrete feedback law $F_{h}$ has been computed numerically following Section 5 .

Also in this example it is in principle possible to derive an explicit formula from the numerical results, it is, however, considerably more complicated, since a number 


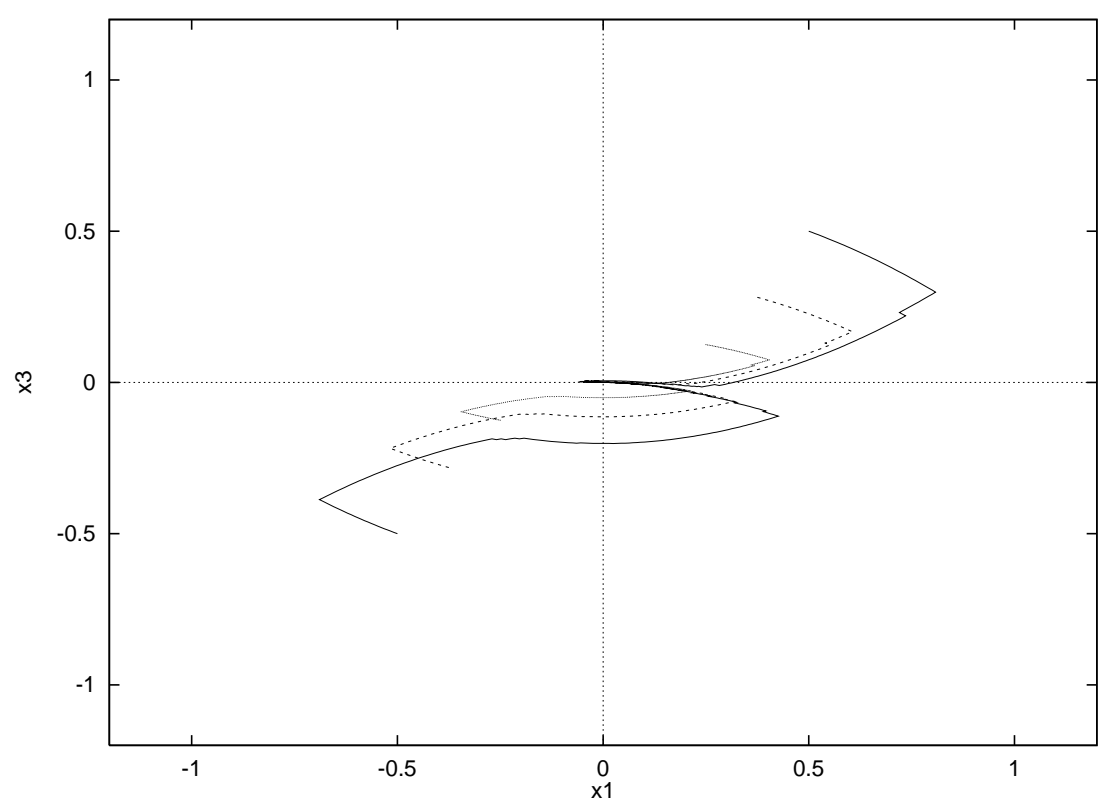

FIG. 7.3. Trajectories for stabilized system (7.2), projected to the $\left(x_{1}, x_{3}\right)$ plane

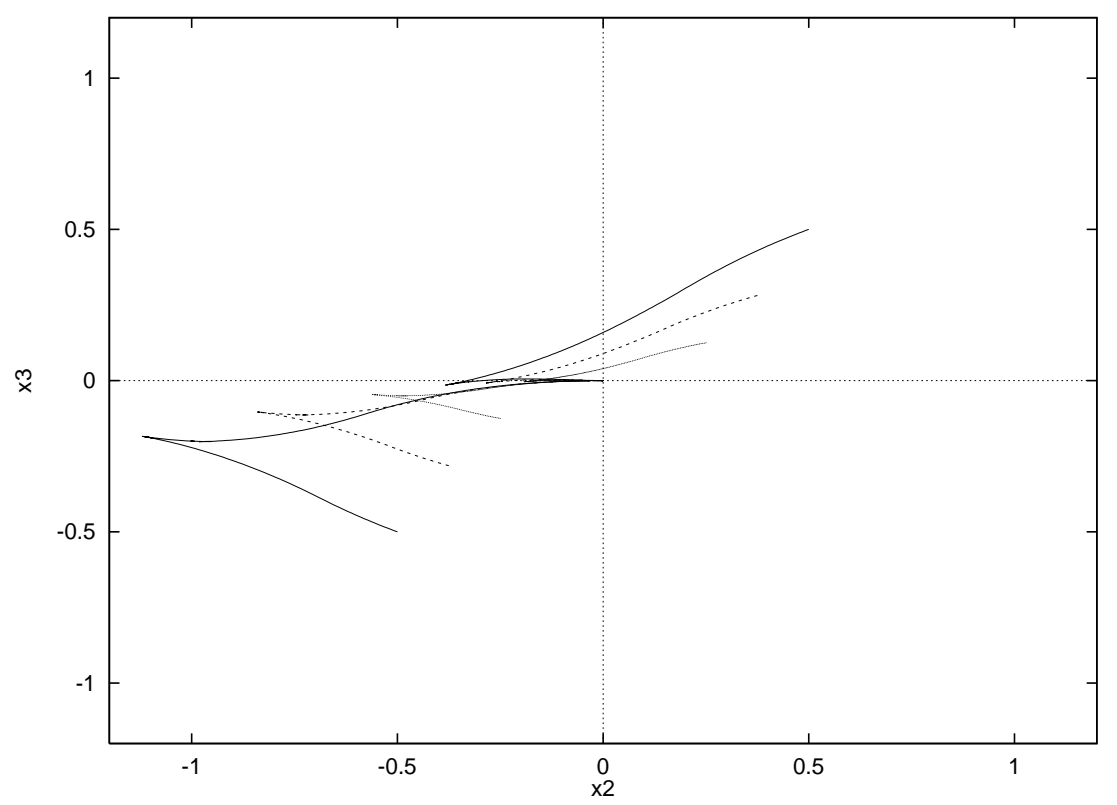

FIG. 7.4. Trajectories for stabilized system (7.2), projected to the $\left(x_{2}, x_{3}\right)$ plane

of switching surfaces have to be identified. Hence we directly used the numerical approximation $F^{h}$ of $F$ for the simulation shown in the Figures 7.2-7.4 in different projections; the time step is $h=0.01$, the controlvalues were chosen as $U=\{-1,1\}$. 


\section{REFERENCES}

[1] M. Bardi and I. Capuzzo Dolcetta, Optimal Control and Viscosity Solutions of HamiltonJacobi-Bellman equations, Birkhäuser, Boston, 1997.

[2] R. Brockett, Asymptotic stability and feedback stabilization, in Differential Geometric Control Theory, R. Brockett, R. Millman, and H. Sussmann, eds., Birkhäuser, Boston, 1983, pp. 181-191.

[3] F. ClARKE, Methods of Dynamic and Nonsmooth Optimization, vol. 22 of CBMS-NSF Regional Conferences Series in Applied Mathematics, SIAM, Philadelphia, 1989.

[4] F. Clarke, Y. Ledyaev, E. Sontag, and A. Subbotin, Asymptotic controllability implies feedback stabilization, IEEE Trans. Autom. Control, 42 (1997), pp. 1394-1407.

[5] F. Colonius and W. Kliemann, Maximal and minimal Lyapunov exponents of bilinear control systems, J. Differ. Equations, 101 (1993), pp. 232-275.

[6] - The Dynamics of Control, Birkhäuser, to appear.

[7] L. GRÜNE, Discrete feedback stabilization of semilinear control systems, ESAIM Control Optim. Calc. Var., 1 (1996), pp. 207-224.

[8] - Numerical stabilization of bilinear control systems, SIAM J. Control Optim., 34 (1996), pp. 2024-2050.

[9] - Discrete feedback stabilization of nonlinear control systems at a singular point, in Proceedings of the 4th European Control Conference, Brussels, 1997. Paper No. 806.

$[10]-$ Asymptotic controllability and exponential stabilization of nonlinear control systems at singular points, SIAM J. Control Optim., 36 (1998), pp. 1585-1603.

[11] _ A uniform exponential spectrum for linear flows on vector bundles, J. Dyn. Differ. Equations, (1999). To appear.

[12] H. Hermes, On stabilizing feedback attitude control, J. Optimization Theory Appl., 31 (1980), pp. 373-384.

[13] - On the synthesis of stabilizing feedback control via Lie algebraic methods, SIAM J. Control Optim., 18 (1980), pp. 352-361.

[14] - Nilpotent and high order approximations of vector field systems, SIAM Rev., 33 (1991), pp. 238-264.

[15] — Homogeneous feedback control for homogeneous systems, System \& Control Lett., 24 (1995), pp. $7-11$.

[16] A. IGGIDR AND J.-C. Vivalda, Global stabilization of homogeneous polynomial systems, Nonlinear Anal., 18 (1992), pp. 1181-1186.

[17] M. KawsKI, Homogeneous feedback stabilization, in New Trends in Systems Theory (Genova, 1990), Progr. Systems Control Theory, vol. 7, Birkhäuser, Boston, 1991, pp. 464-471.

[18] Y. Ledyaev and E. Sontag, A Lyapunov characterization of robust stabilization, J. Nonlinear Anal. To appear.

[19] L. Rosier, Homogeneous Liapunov function for continuous vector fields, System \& Control Lett., 19 (1992), pp. 467-473.

[20] E. RYAN, Universal stabilization of a class of nonlinear systems with homogeneous vector field, System \& Control Lett., 26 (1995), pp. 177-184.

[21] R. Sepulchre And D. Ayels, Homogeneous Lyapunov functions and necessary conditions for stabilization, Math. Control Signals Systems, 9 (1996), pp. 34-58.

$[22]$ - Stabilizability does not imply homogeneous stabilizability for controllable homogeneous systems, SIAM J. Control Optim., 34 (1996), pp. 1798-1813.

[23] E. SonTaG, Nonlinear regulation: The piecewise linear approach, IEEE Trans. Autom. Control, AC-26 (1981), pp. 346-358.

[24] - Stability and stabilization: Discontinuities and the effect of disturbances, in Proc. NATO Advanced Study Institute "Nonlinear Analysis, Differential Equations, and Control" (Montreal, Jul/Aug 1998), Kluwer, 1999.

[25] J. Tsinias, Remarks on feedback stabilizability of homogeneous systems, Control Theory Adv. Tech., 6 (1990), pp. 533-542. 